Research Paper

\title{
In Vitro Assessment of the Gene Expression of EZH-2 and P300 During Motor Neuron Differentiation of Human Umbilical Cord Blood Mesenchymal Stem Cells
}

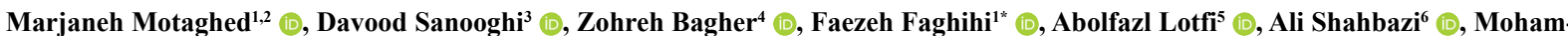 \\ mad Taghi Jogataei ${ }^{1^{*}}$ \\ 1. Cellular and Molecular Research Center, Iran University of Medical Sciences, Tehran, Iran. \\ 2. International Campus, Iran University of Medical Sciences, Tehran, Iran. \\ 3. Department of Genetics, School of Biological Sciences, Shahid Beheshti University, Tehran, Iran. \\ 4. ENT and Head and Neck Research Center and Department, Five Senses Institute, Hazrat Rasoul Akram Hospital, Iran University of Medical Sciences, \\ Tehran, Iran. \\ 5. Damavand Agricultural College, Technical and Vocational University, Tehran, Iran. \\ 6. Department of Neuroscience, School of Advanced Technologies in Medicine, Iran University of Medical Sciences, Tehran, Iran.
}

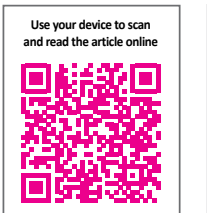

Citation Motaghed, M., Sanooghi, D., Bagher, Z., Faghihi, F., Lotfi, A., Shahbazi, A., et al. (2022). In Vitro Assessment of the Gene Expression of EZH-2 and P300 During Motor Neuron Differentiation of Human Umbilical Cord Blood Mesenchymal Stem Cells. Basic and Clinical Neuroscience, 13(5), 709-718. http://dx.doi.org/10.32598/bcn.2021.2997.1.

iol http://dx.doi.org/10.32598/ben.2021.2997.1

Article info:

Received: 04 Nov 2020

First Revision: 10 Mar 2021

Accepted: 01 Apr 2021

Available Online: 01 Sep 2022

Keywords:

Cord blood, Motor Neuron, P300, EZH-2

\section{$\underline{\text { A B S T RA C T }}$}

Introduction: Maintenance of neurogenesis depends on the function of somehistone-modifying enzymes; including Enhancer of zeste homolog 2 (EZH2) and histone acetyltransferases (P300). The mechanism of epigenetic regulation and gene expression underlying the transition of human umbilical cord blood mesenchymal stem cells (hUCB-MSCs) into MNs has not been fully clarified.

Methods: Two morphogens; sonic hedgehog (Shh: $100 \mathrm{ng} / \mathrm{mL}$ ) and retinoic acid (RA: 0.01 $\mathrm{mM}$ ) were involved in the specification of hUCB-MSCs into MNs after MSC characterization using Flow cytometry. Real time-quantitative PCR and immunocytochemistry were performed to find the expression of the genes at the level of mRNA and protein.

Results: The expression of MN-related markers was confirmed at the level of mRNA and protein by induction of differentiation. The results were confirmed by immunocytochemistry and showed those mean cell percentages of $55.33 \% \pm 15.885 \%$ and $49.67 \% \pm 13.796 \%$ could express Islet-1 and ChAT, respectively. The gene expression level of Islet-1 and ChAT was significantly increased in the first and second week of exposure, respectively. After two weeks, the expression level of P300 and EZH-2 genes increased remarkably. No significant expression of Mnx-1 was detected when compared to the control sample.

Conclusion: MN-related markers, Islet-1 and ChAT, were detected in differentiated cells of hUCB-MSCs, supporting the potency of cord blood cells in the regeneration of MN-related disorders. Assessing these epigenetic regulatory genes at the protein level can be suggested to confirm their functional epigenetic modifying effects during motor neuron differentiation.

\footnotetext{
* Corresponding Author:

Faezeh Faghihi, PhD.

Address: Cellular and Molecular Research Center, Iran University of Medical Sciences, Tehran, Iran.

E-mail:faezefaghihi@yahoo.com
} 


\section{Highlights}

- The overexpression of P300 along with ChAT in differentiating motorneuron like cells

- The overexpression EZH2 along with ChAT in differentiating motorneuron like cells

- The expression of motorneuron- related genes in differentiating hUC-MSCs.

\section{Plain Language Summary}

Generation of motor neuron(MN) cells in human nervous system depends on genes and enzymes; such as some histone- modifying enzymes; like Enhancer of zeste homolog 2 (EZH2) and histone acetyltransferases (P300). Stem cells derived from human umbilical cord blood (hUCB- MSCs) are potent to differentiate MN cells and using in regeneration of damaged neural cells. In this study we explored whether we could detect the expression of MN-related genes, along with $\mathrm{P} 300$ and EZH2 as modulating genes in MNs derived from human UCB stem cells.

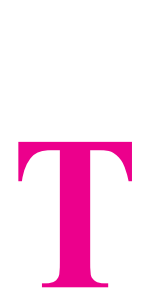

\section{Introduction}

he distinction of stem cells into a certain type of cell requires the upregulation of genes that have an important role in a special phenotype and suppression of the other genes. Because of these imposed alterations, histone-modifying enzymes can regulate the accessibility of transcription factors and other modulators to the genes (Vincent \& Van Seuningen, 2009; Qiu, 2006). It has been stated that the Enhancer of Zeste Homolog 2 (EZH2), known as an evolutionarily conserved gene, controls the transference from proliferation phase to differentiation (Gan et al., 2018), accelerates, and maintains the onset of neurogenesis (Wever et al., 2019). Previous studies demonstrate that the equivalency in two processes of differentiation and regeneration in the cerebral cortex can be regulated by EZH-2 (Pereira et al., 2010). It controls the neural stem cell state (Rai et al., 2010) and prevents premature differentiation (Pereira et al., 2010; Lilja et al., 2013). Another histone-modifying protein, P300, contributes to the maintenance of the expression of Islet-1 as an early motor neuron marker in the development of embryonic spinal cord motor neurons (Liang et al., 2011; Toch \& Clotman, 2019; Lee et al., 2009; Partanen et al., 2004). Based on these reports, the expression of motor neuron genes decrease in the mutant embryos with P300 deficiency. It suggests that excessive function of P300 in neural progenitors may accelerate $\mathrm{MN}$ specification during the nervous system development.
Moreover, the involvement of Sonic Hedgehog (Shh) and retinoic acid (RA) in MN specification has also been stated (Novitch et al., 2003; Lee \& Pfaff, 2003). The administration of these ingredients increases the presence of markers involved in MNs. The significant role of RA in the developmental conversion of stem cells into neural ancestries has been stated. However, the mechanisms for suppressing RA-regulated genes have remained unclear (Urvalek \& Gudas, 2014).

Furthermore, the hedgehog family is known as evolutionary conserved developmental morphogens, which have a vital role in the function and development of stem cells (Fattahi et al., 2018). For example, it has been reported that Shh signaling executes positive regulation on the expression of EZH-2, which is introduced as an evolutionarily conserved gene via an epigenetic mechanism. It can manage the expression of some genes involved in several biological functions (Gan et al., 2018).

Until now, the mechanisms underlying the epigenetic rules and expression of genes responsible for the conversion of human umbilical cord blood mesenchymal stem cells (hUCB-MSCs) into MNs have not been reported clearly. Therefore, this study quantitatively investigates the expression of MN genes (ChAT, Islet-1, and Mnx-1), along with the two effective genes (EZH2 and P300, which are included between regulatory histone methyltransferase genes and histone acetyltransferase genes, respectively) to control epigenetic changes during shifting of hUCB-MSCs into MNs, using Shh and RA. These two morphogens can control $\mathrm{MN}$ development and the specification of the spinal cord (Novitch, et al., 2003; Lee \& Pfaff, 2003). 


\section{Materials and Methods}

Isolation and culture of human umbilical cord blood mesenchymal stem cells

Human umbilical cord blood mesenchymal stem cells were collected from the umbilical vein of the neonates after elective cesarean sections. Following the preparation of parental informed consent, the Ethics Committee of Iran University of Medical Sciences approved using these cells. The isolation of hUCB-MSCs was done according to the protocol mentioned in our first reports (Yousefi et al., 2017). Briefly, $10 \mu \mathrm{L}$ of heparin was added into a $50-\mathrm{mL}$ sterile syringe containing blood samples. Within 4 hours after sample collection, red blood cells were removed using about $1 \mathrm{~mL}$ of $10 \%$ hydroxyethyl starch (Fresenius, Germany). The supernatant was collected and diluted in phosphate-buffered saline, supplemented with penicillin $(100 \mu \mathrm{g} / \mathrm{mL}) /$ streptomycin $(0.1$ $\mathrm{mg} / \mathrm{mL}$ ) (Sigma, USA), $15 \%$ fetal bovine serum (Gibco, Germany), and $0.2 \%$ Ethylenediaminetetraacetic acid (EDTA). The mononuclear cells were collected doing centrifugation for $30 \mathrm{~min}$ at $1200 \mathrm{rpm}$ with a ratio of 1:3 over Ficoll-hypaque (Inno-Train, Germany). The cells were washed with PBS (phosphate buffered saline), then cultured in culture flasks $\left(75 \mathrm{~cm}^{2}\right)$ containing DMEMF12 supplemented with penicillin/streptomycin and 10\% FBS. The flasks were incubated at $37^{\circ} \mathrm{C}$ and $5 \% \mathrm{CO}_{2}$. The cells were put into new flasks when they were filled with $70 \%$ confluent cells, and the medium was also renewed every 3 days. The cells after three passages were applied in the experiments.

\section{Characterization of hUCB-MSCs}

The characterization of MSCs was done using monocolor cytofluorimetric analysis. About 105 cells were cultured in $10 \%$ goat serum at $4^{\circ} \mathrm{C}$. The serum was separated from the cells after one hour. Again, cells were incubated for $40 \mathrm{~min}$ at $4^{\circ} \mathrm{C}$ with fluorescein isothiocyanate and phycoerythrin-conjugated monoclonal antibodies against human CD90, CD73, CD45, CD44, and CD34 (BD bioscience, USA). To detect the expression of the antigens, the flow-cytometry studies (BectonDickinson) were done using FlowJo software. For the control sample, isotype-matched antibodies were used.

\section{Induction of hUCB-MSCs into MN-like cells}

According to the protocol of the previous study (Faghihi et al., 2015; Faghihi et al., 2016), the induction of hUCBMSCs into MN-like cells was done. In summary, a 24well plate containing approximately $2 \times 105$ cells was incubated overnight at $37^{\circ} \mathrm{C}$ with $5 \% \mathrm{CO}_{2}$. For the following day, the cells remained in a humidified incubator again for another overnight after the replacement of the expansion medium with the new medium (DMEM-F12), which consisted of $20 \%$ FBS, $0.2 \%$ B27 (Gibco, Germany), basic fibroblast growth factor with the concentration of $10 \mathrm{ng} / \mathrm{mL}$ (Sigma, USA), $\beta$-mercaptoethanol (100mM), and isobutylmethylxanthine $(250 \mathrm{mM})$. On the next day, the cells were exposed to the fresh medium, added with Shh $(100 \mathrm{ng} / \mathrm{mL})$ (R\&D, USA), RA (0.01mM) (Sigma, USA), and $0.2 \%$ B27. After one week, the brain-derived neurotrophic factor with a concentration of $100 \mathrm{ng} / \mathrm{mL}$ (Invitrogen, USA) and $0.2 \%$ B27 was added to the fresh medium for another week.

\section{Immunocytochemical staining}

After two weeks, the cells were fixed in $4 \%$ paraformaldehyde for the process of immunostaining to reveal the expression of related antigens. Permeabilization of cells using $0.2 \%$ Triton X-100 was done at room temperature. The cells were washed with PBS and incubated at room temperature for 60 min with $10 \%$ goat serum. Afterward, the cells were raised with primary antibodies against human ChAT (Abcam, USA), Mnx-1 (Abcam, USA), and Islet-1 (Santacruz, USA). Then the cells were incubated with PE and FITCconjugated secondary antibodies at $37^{\circ} \mathrm{C}$ for $45 \mathrm{~min}$. The cell nuclei were stained with DAPI (Sigma, USA) and observed using a fluorescent microscope (Olympus DP70, Japan). The cells from ten unbiased fields were counted for each antibody, and the results were reported as Mean \pm SE.

\section{Gene expression analysis by RT-qPCR}

Real time-quantitative PCR was performed to authenticate the expression of both MN-related genes and the regulatory epigenetic genes (P300 and EZH2). After post-induction days, the cells were trypsinized with Trypsin-EDTA (0.25\%). Total RNA was extracted using TRIzol (Invitrogen, Germany). DNase I was used to digest DNA, and then the purity of mRNA was found spectrometrically. The cDNA synthesis kit (Fermentas, Canada) was used to synthesize the cDNA from mRNA. The real-time qPCR reactions were performed in reaction plates using Applied Biosystems, USA. Three separate mRNA samples were used. A mixture of $1 \times \mathrm{SYBR}^{\circledR}$ Green PCR Master Mix with approximately $12 \mathrm{ng}$ of obtained cDNA and its equivalent primers (Table 1) was prepared with a total volume of $20 \mathrm{mM}$ for each reaction. The fold changes of genes were identified after normalization of the expression level of genes to the internal control, which was GAPDH in this study. 
Table 1. List of primers used in RT-qPCR

\begin{tabular}{cc}
\hline Target Gene & The Forward and Reverse Sequence of Primers \\
\hline GAPDH & F: CTCATTTCCTGGTATGACAAC \\
R: CTTCCTCTTGTGCTCTTGCT & F: GCA GGAGAAGACAGCCAACT \\
R: TGCAAACCTCAGCTGGTCAT & F: AGCACCAGTTCAAGCTCAACA \\
Mnx-1 & R: ACCAATCTTCACCTGGGTCTC \\
Islet-1 & F: ATATCAGGTTGTACGGGATCAAATG \\
& R: CACGCATCACGAAGTCGTTC \\
P300 & F: GTTCTCCTTACAGCAGCAACA \\
R: GCAGAGGATTCATGTTCTGCAAG \\
\hline EZ-2 & F: CCGGGCTAGGTTAATTGGGACCAAA \\
\hline
\end{tabular}

\section{Results}

\section{Isolation and characterization of hUCB-MSCs}

The isolated MSCs from human umbilical cord blood exhibited spindle shape morphology at passage three (Figure 1a). These cells could express CD44, CD73, and CD90 markers on their surfaces, but they could not express hematopoietic antigens, such as CD45 and CD34 (Figure 1b).

\section{Differentiation of hUCB-MSCs}

There were some dead cells due to the induction of hUCBMSCs with Shh and RA, but the remaining cells showed the ability to express MN-related markers. Immunochemical staining revealed that the cells had no expression of Mnx1 but could express $53 \% \pm 10.148 \%$ and $45 \% \pm 13.453 \%$ of Islet-1 and ChAT, respectively (Figure 2).

\section{Statistical Analysis of Gene Expression}

The analysis results demonstrated that the induction of hUCB-MSCs with the specific induction medium could lead to the overexpression of Islet-1, ChAT, and MN-related genes, after one or two week(s) of exposure with a significant $\mathrm{P}$ value of $<0.05$. Consequently, the overexpression level of histone modifying genes
P300 and EZH-2, compared to the control samples (not exposed cells), were also found after two weeks, significantly with the $\mathrm{P} \leq 0.05$ (Figure 3 ).

\section{Discussion}

In regenerative medicine, using the human cord blood mesenchymal stem cells (hUCB-MSCs) due to their immunomodulatory properties and their capacity for neural differentiation (Qiao et al., 2008; Romanov, Svintsitskaya, \& Smirnov, 2003) is a valuable tool for stem cell studies which was used in this study as well. Generally, the differentiation tendency in cells can be affected by various epigenetic factors. This condition can lead to less differentiation capacity and specific cell type distinction (Keller et al., 2018).

The obtained results from this study showed that the differentiated hUCB-MSCs could express MN-specific genes, including choline acetyltransferase (ChAT) and Islet-1, at the level of mRNA and protein. Previous studies reported that the combination of Shh and RA morphogens upregulates the expression of ChAT and Islet-1 (Park et al., 2012; Thaler et al., 2004). Islet-1 has been introduced as a transcription factor that has a prominent role in the differentiation of cholinergic amacrine cells and supports the differentiation and survival of MNs

Table 2. The quantitative results of immunocytochemistry

\begin{tabular}{cccc}
\hline Plate & Islet-1 & ChAT & Mnx-1 \\
\hline 1 & 55 & 60 & Not detected \\
2 & 42 & 34 & Not detected \\
3 & 62 & 41 & Not detected \\
Mean \pm SD & $53 \pm 10.148$ & $45 \pm 13.453$ & Not detected
\end{tabular}




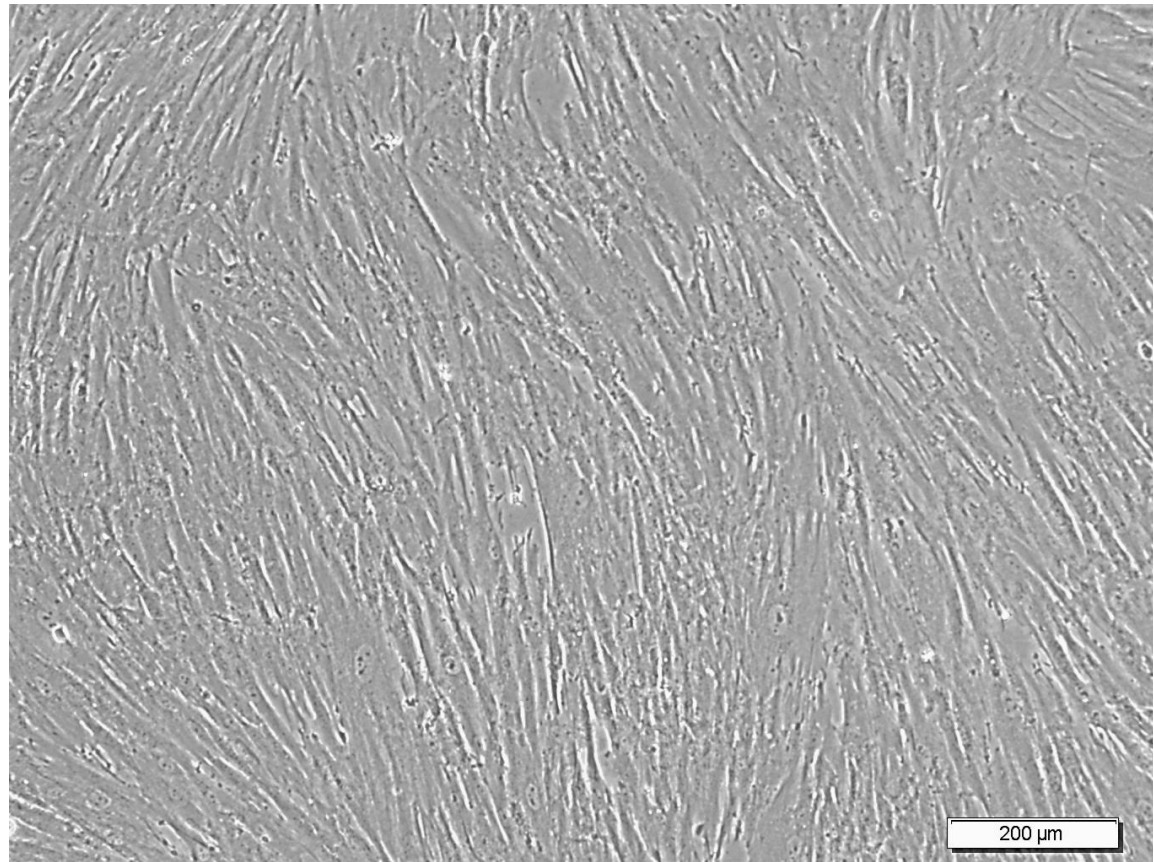

Figure 1. Human Umbilical Cord Blood-Mesenchymal Stem Cells

NEUR:SCIENCE

a) Cord blood-mesenchymal stem cells (magnification $\times 4$ ) with fibroblast-like shape are shown in passage \#3. b) Cytofluorimetric analysis of the isolated cells in passage \#3 revealed that they express antigens CD90, CD73, and CD44, while leukocyte and hematopoietic markers, including CD45 and CD34, could not be expressed.

(Galli-Resta et al,. 1997). We could detect a significant overexpression of the Islet-1 gene (early motor neuron marker) and ChAT gene (late motor neuron marker) at the end of the first week (after exposure to Shh and RA) and the end of the second week (without exposure to Shh and RA). The findings showed that upon the differentiation stage, the Mnx-1 expression was slightly increased while there were no significant changes in its mRNA lev-
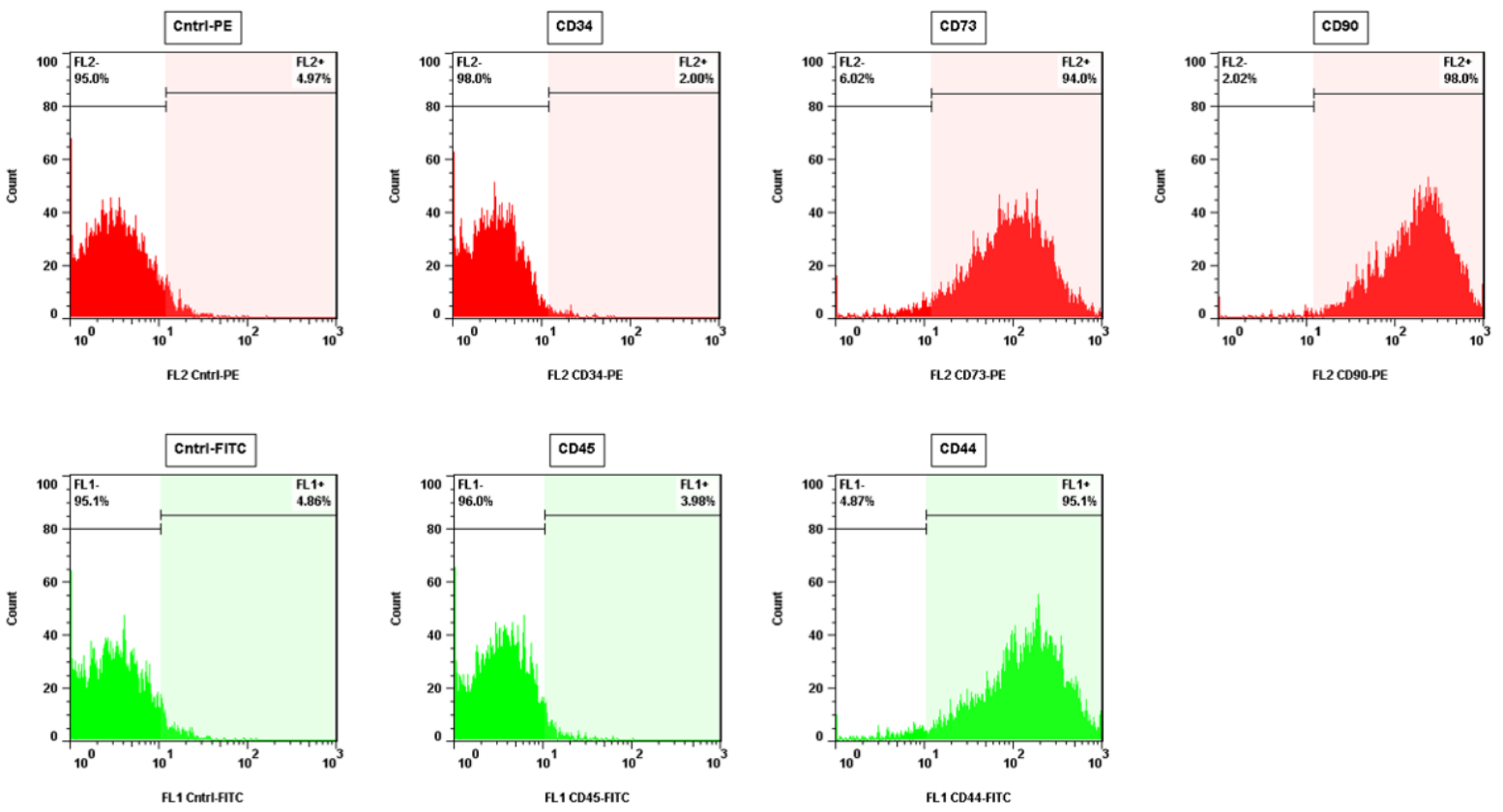

NEUR SCIENCE

Figure 2. Immunocytochemistry on Motor Neuron (MN)-like Cells Derived From Human Umbilical Cord Blood-Mesenchymal Stem Cells

The expression of antigens Islet- $1(55.33 \% \pm 15.88 \%)$, ChAT $(49.67 \% \pm 13.796 \%)$, and Mnx-1 were found after two weeks of induction. 


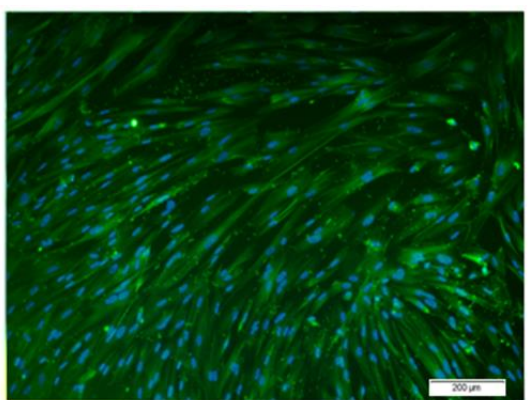

Islet-1

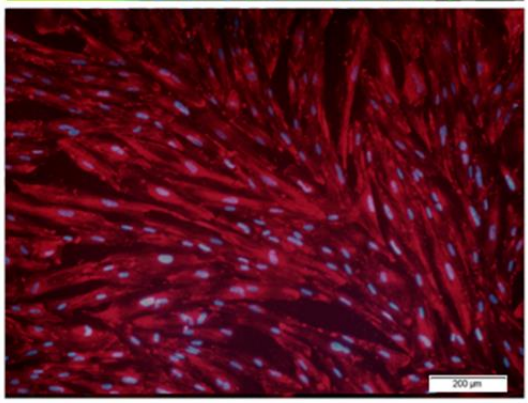

ChAT

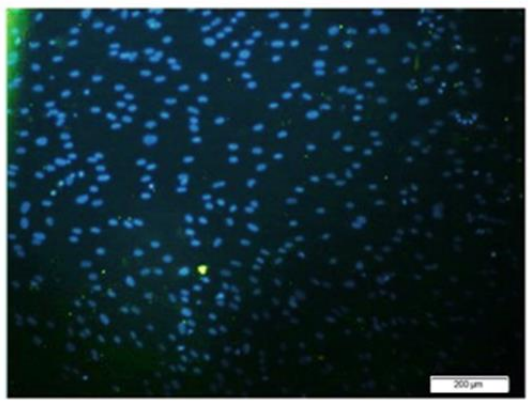

\section{$\operatorname{Mnx}-1$}

NEUR SCIENCE

Figure 3. Quantitative Evaluation of the Expression of Motor Neurons (MN)-related Genes and Histone Modifying Enzymes

The expression of ChAT, Islet-1, and Mnx-1 was found in human cord blood-mesenchymal stem cells 7 and 14 days post-induction $(\mathrm{P}<0.05)$. The MN-related genes, Islet-1, ChAT, and histone-modifying enzymes, P300 and EZH-2 were upregulated significantly after two weeks when compared with the control sample (non-treated CB-MSCs) and the cells at the first week ( $\mathrm{P} \leq 0.05)$. The expression of Mnx1 increased slightly after the second week, while it was not significant.

el or protein. Moreover, the silencing of the Mnx-1 gene in mice at the early stages of development showed that the number of degenerated MNs reduced abnormally. Furthermore, the downstream genes of Islet-1 remained unaffected in the absence of Mnx-1 (Liang et al., 2011).

The expression of P300 and EZH-2 was found after two weeks in the absence of Shh and RA. Even though the regulatory role of EZH-2 in the transition of cells from proliferation to differentiation has been identified, it showed an ability to suppress forebrain activities
(Zemke et al., 2015). It was also shown that conditional removal of EZH-2 before the onset of neurogenesis in cortical progenitors shifted the cells toward differentiation and maturation (Pereira et al., 2010; Pasini et al, 2007; O'Carroll et al,. 2001). In our study, the upregulation of EZH-2 could be due to the tendency of morphogen-inducible markers to induce the cells in an immature state as early motor neurons. It has also been reported that the deletion of EZH-2 at a later time point in neural precursors results in an increased number of neurons ( $\mathrm{Pi}-$ etersen \& van Lohuizen, 2008). Accordingly, these reported data pointed out the severe time-dependent role of EZH2, which has transactions in neurogenesis (Pereira et al., 2010).

Moreover, the overexpression of the P300 gene was found after two weeks following the removal of Shh and RA. Previous studies show the necessity of the P300 gene in developing astrocytes and regeneration of axons (Cheng, 2011; Lilja et al., 2013). MNs in the region of the spinal cord can be regulated with P300 during neuronal differentiation and neurogenesis along with CREB binding protein (CBP) (Lee et al., 2009; Partanen, Motoyama, \& Hui, 2004). Furthermore, the synergistic interaction of RA receptor and Neurogenin-2 has been reported in developing MNs involved in the spinal cord (Lee et al., 2009). The contribution of P300 and CBP in the expression of Islet-1 in embryonic spinal motor neurons was identified (Toch \& Clotman, 2019). As we could see, the expression of Islet-1, an important transcription factor involved in cholinergic differentiation, was upregulated after induction with RA and Shh. The Islet-1 gene has been reported to be necessary for developing motor neurons (Pfaff, Mendelsohn, Stewart, Edlund, \& Jessell, 1996).

A reduction in the number of MNs was reported in the P300/CBP compound obtained from mutant embryos, suggesting the important role of these two activators in neural progenitors in promoting MN specification (Toch, \& Clotman, 2019). However, another study suggests the function of P300/CBP in postmitotic MNs to control later aspects of development (Lee et al., 2009). Moreover, the low expression of Islet-1 in MNs, suggests that the postmitotic MN activity of P300/CBP may depend on the expression of Islet-1 (Toch, \& Clotman, 2019).

In this study, MNs-related markers, Islet-1 and ChAT, were detected in differentiated hUCB-MSCs, supporting the potency of cord blood cells which might be used in the renewal of MN-related genes damaged in disorders. We could also detect the upregulation of Islet-1 as an early MN marker in the presence of Shh 

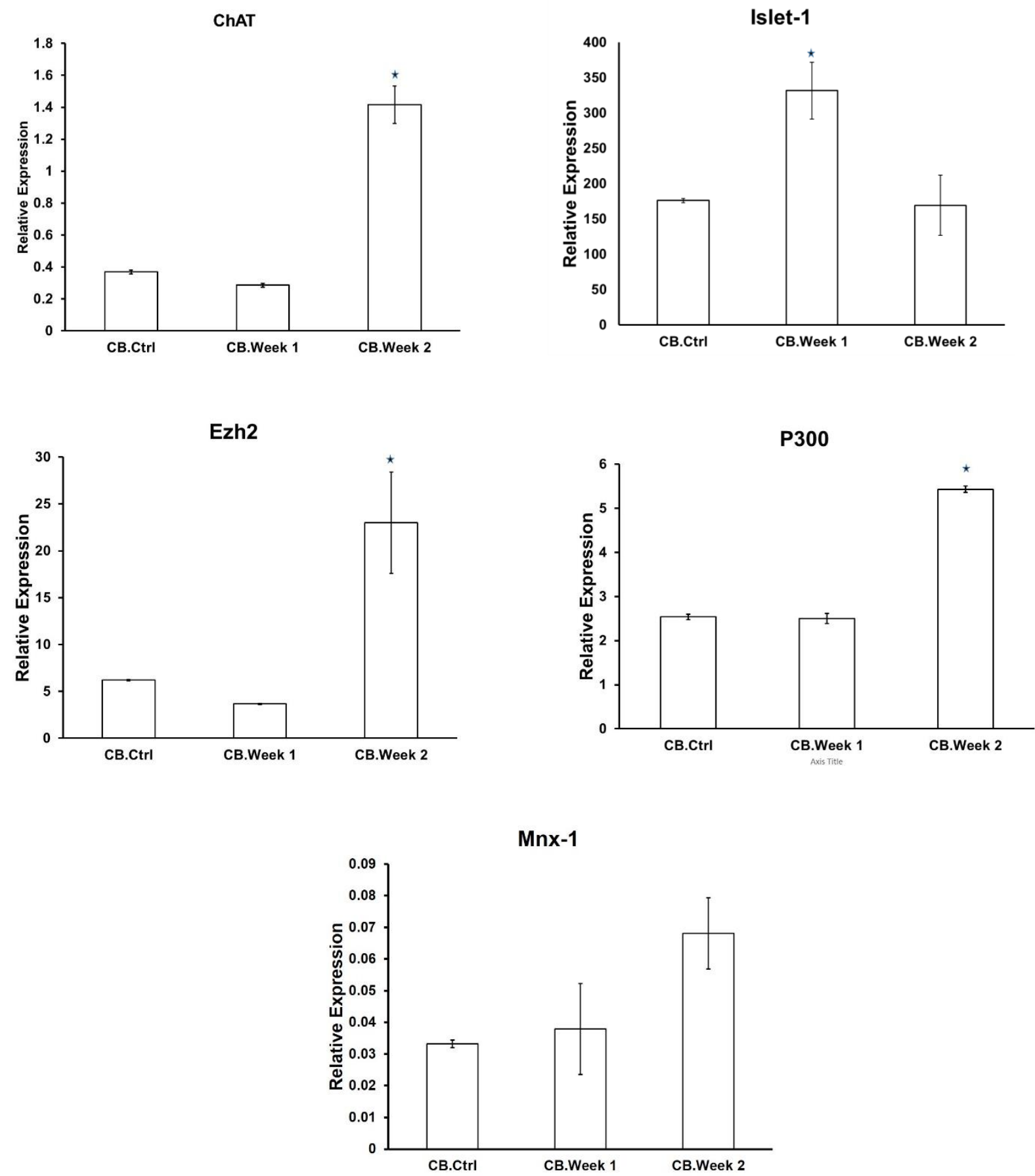

Figure 4. Relative Expression of Un-exposed Cells, ChAT, Islet-1, Mnx-1, P300, and EZH-2 at week 1 and week 2

and RA, which indicates the supportive effect of these morphogens for the onset of motor neuron generation. We could also detect remarkable overexpression of $\mathrm{P} 300$ and $\mathrm{EZH} 2$, known as epigenetic regulatory genes involved in neurogenesis, along with ChAT as the mature motor neuron marker after two weeks following the removal of Shh and RA. To our knowledge, the expression of $\mathrm{P} 300$ and $\mathrm{EZH}-2$ during the differentiation of hUCB-MSCs into MN-like cells was performed in this study for the first time. However, the expression of these epigenetic regulatory genes at the protein level must be evaluated to confirm their epigenetic modifying effects during motor neuron differentiation. 


\section{Ethical Considerations}

\section{Compliance with ethical guidelines}

The collection of human umbilical cord blood for isolation of mesenchymal stem cells has been done after obtaining mothers informed consent. All the protocols and the procedures in this project were approved by the ethical committee of Iran University of medical Sciences (IUMS) (Code: IR.IUMS.REC 1395.95-03-117-28604).

\section{Funding}

This study was funded by IIran University of medical Sciences (IUMS) (Grant No.: 95-03-117-28604) and from Iran National Science Foundation and Pad Nahad Tabiat Co. Ltd (Grant No.: 95835564).

\section{Authors' contributions}

All authors equally contributed to preparing this article.

\section{Conflict of interest}

The authors declared no competing interest.

\section{Acknowledgments}

The authors would like to appreciate the staff of Cellular and Molecular Research Center.

\section{References}

Cheng, P. Y., Lin, Y. P., Chen, Y. L., Lee, Y. C., Tai, C. C., \& Wang, Y. T., et al. (2011). Interplay between SIN3A and STAT3 mediates chromatin conformational changes and GFAP expression during cellular differentiation. PLoS One, 6(7), e22018. [PMID] [PMCID]

Faghihi, F., Mirzaei, E., Ai, J., Lotfi, A., Sayahpour, F. A., \& Barough, S. E., et al. (2016). Differentiation potential of human chorion-derived mesenchymal stem cells into motor neuron-like cells in two-and three-dimensional culture systems. Molecular Neurobiology, 53(3), 1862-1872. [PMID]

Faghihi, F., Mirzaei, E., Sarveazad, A., Ai, J., Ebrahimi Barough, S., \& Lotfi, A., et al. (2015). Differentiation potential of human bone marrow mesenchymal stem cells into motorneuron-like cells on electrospun gelatin membrane. Journal of Molecular Neuroscience, 55(4), 845-853. [PMID]

Fattahi, S., Pilehchian Langroudi, M., \& Akhavan-Niaki, H. (2018). Hedgehog signaling pathway: Epigenetic regulation and role in disease and cancer development. Journal of Cellular Physiology, 233(8), 5726-5735. [PMID]
Galli-Resta, L., Resta, G., Tan, S. S., \& Reese, B. E. (1997) Mosaics of islet-1-expressing amacrine cells assembled by short-range cellular interactions. The Journal of Neuroscience : The Official Journal of The Society for Neuroscience, 17(20), 7831-7838.[PMID] [PMCID]

Gan, L., Yang, Y., Li, Q., Feng, Y., Liu, T., \& Guo, W. (2018). Epigenetic regulation of cancer progression by EZH2: From biological insights to therapeutic potential. Biomarker Research, 6, 10. [PMID]

Keller, A., Dziedzicka, D., Zambelli, F., Markouli, C., Sermon K., \& Spits, C., et al.(2018). Genetic and epigenetic factors which modulate differentiation propensity in human pluripotent stem cells. Human Reproduction Update, 24(2), 162-175. [PMID]

Lee, S. K., \& Pfaff, S. L. (2003). Synchronization of neurogenesis and motor neuron specification by direct coupling of bHLH and homeodomain transcription factors. Neuron 38(5), 731-745. [PMID]

Lee, S., Lee, B., Lee, J. W., \& Lee, S. K. (2009). Retinoid signaling and neurogenin 2 function are coupled for the specification of spinal motor neurons through a chromatin modifier CBP. Neuron, 62(5), 641-654. [PMID] [PMCID]

Liang, X., Song, M. R., Xu, Z., Lanuza, G. M., Liu, Y., \& Zhuang, T., et al. (2011). Isl1 is required for multiple aspects of motor neuron development. Molecular and Cellular Neuroscience, 47(3), 215-222. [PMID] [PMCID]

Lilja, T., Heldring, N., \& Hermanson, O. (2013). Like a rolling histone: Epigenetic regulation of neural stem cells and brain development by factors controlling histone acetylation and methylation. Biochimica et Biophysica Acta, 1830(2), 2354-2360. [PMID]

Novitch, B. G., Wichterle, H., Jessell, T. M., \& Sockanathan, S (2003). A requirement for retinoic acid-mediated transcriptional activation in ventral neural patterning and motor neuron specification. Neuron, 40(1), 81-95. [PMID]

O'Carroll, D., Erhardt, S., Pagani, M., Barton, S. C., Surani, M A., \& Jenuwein, T. (2001). The polycomb-group geneEzh2 is required for early mouse development. Molecular and Cellular Biology, 21(13), 4330-4336. [PMID] [PMCID]

Park, H. W., Cho, J. S., Park, C. K., Jung, S. J., Park, C. H., \& Lee, S. J., et al. (2012). Directed induction of functional motor neuron-like cells from genetically engineered human mesenchymal stem cells. PloS One, 7(4), e35244. [PMID] [PMCID]

Partanen, A., Motoyama, J., \& Hui, C. C. (1999). Developmentally regulated expression of the transcriptional cofactors/ histone acetyltransferases CBP and p300 during mouse embryogenesis. The International Journal of Developmental Biology, 43(6), 487-494. [PMID]

Pasini, D., Bracken, A. P., Hansen, J. B., Capillo, M., \& Helin K. (2007). The polycomb group protein Suz12 is required for embryonic stem cell differentiation. Molecular and Cellular Biology, 27(10), 3769-3779. [PMID] [PMCID]

Pereira, J. D., Sansom, S. N., Smith, J., Dobenecker, M. W., Tarakhovsky, A., \& Livesey, F. J. (2010). Ezh2, the histone methyltransferase of PRC2, regulates the balance between self-renewal and differentiation in the cerebral cortex. Proceedings of the National Academy of Sciences, 107(36), 15957-15962. [PMID] 
Pietersen, A. M., \& van Lohuizen, M. (2008). Stem cell regulation by polycomb repressors: Postponing commitment. Current Opinion in Cell Biology, 20(2), 201-207. [PMID]

Qiao, C., Xu, W., Zhu, W., Hu, J., Qian, H., \& Yin, Q., et al (2008). Human mesenchymal stem cells isolated from the umbilical cord. Cell Biology International, 32(1), 8-15. [PMID]

Qiu, J. (2006). Epigenetics: unfinished symphony. Nature, 441(7090), 143-145. [PMID]

Romanov, Y. A., Svintsitskaya, V. A., \& Smirnov, V. N. (2003). Searching for alternative sources of postnatal human mesenchymal stem cells: Candidate MSC-like cells from umbilical cord. Stem Cells, 21(1), 105-110. [PMID]

Rai, K., Jafri, I. F., Chidester, S., James, S. R., Karpf, A. R., \& Cairns, B. R., et al. (2010). Dnmt3 and G9a cooperate for tissue-specific development in zebrafish.The Journal of Biological Chemistry, 285(6), 4110-4121. [PMID]

Thaler, J. P., Koo, S. J., Kania, A., Lettieri, K., Andrews, S., \& Cox, C., et al. (2004). A postmitotic role for Isl-class LIM homeodomain proteins in the assignment of visceral spinal motor neuron identity. Neuron, 41(3), 337-350. [PMID]

Toch, M., \& Clotman, F. (2019). CBP and p300 coactivators contribute to the maintenance of Isl1 expression by the Onecut transcription factors in embryonic spinal motor neurons. Molecular and Cellular Neuroscience, 101, 103411. [PMID]

Urvalek, A. M., \& Gudas, L. J. (2014). Retinoic acid and histone deacetylases regulate epigenetic changes in embryonic stem cells. The Journal of Biological Chemistry, 289(28), 19519 19530.[PMID] [PMCID]

Vincent, A., \& Van Seuningen, I. (2009). Epigenetics, stem cells and epithelial cell fate. Differentiation; Research in Biological Diversity, 78(2-3), 99-107. [PMID]

Wever, I., Wagemans, C., \& Smidt, M. P. (2019). EZH2 is essential for fate determination in the mammalian Isthmic area. Frontiers in Molecular Neuroscience, 12, 76. [PMID] [PMCID]

Yousefi, B., Sanooghi, D., Faghihi, F., Joghataei, M. T., \& Latifi, N. (2017). Evaluation of motor neuron differentiation potential of human umbilical cord blood-derived mesenchymal stem cells, in vitro. Journal of Chemical Neuroanatomy, 81, 18-26. [PMID]

Zemke, M., Draganova, K., Klug, A., Schöler, A., Zurkirchen, L. \& Gay, M. H., et al. (2015). Loss of Ezh2 promotes a midbrainto-forebrain identity switch by direct gene derepression and Wnt-dependent regulation. BMC Biology, 13, 103. [PMID] 
This Page Intentionally Left Blank 